\title{
DriveID: safety innovation through individuation
}

\author{
Ben Sawyer ${ }^{\mathrm{a},}$, Grace Teo ${ }^{\mathrm{a}}$ and Mustapha Mouloua ${ }^{\mathrm{a}}$ \\ ${ }^{\mathrm{a}}$ Department of Psychology, University of Central Florida, 4000 Central Florida Boulevard, Orlando, FL 32816, \\ United States of America
}

\begin{abstract}
The driving task is highly complex and places considerable perceptual, physical and cognitive demands on the driver. As driving is fundamentally an information processing activity, distracted or impaired drivers have diminished safety margins compared with non- distracted drivers (Hancock and Parasuraman, 1992; TRB 1998 a \& b). This competition for sensory and decision making capacities can lead to failures that cost lives. Some groups, teens and elderly drivers for example, have patterns of systematically poor perceptual, physical and cognitive performance while driving. Although there are technologies developed to aid these different drivers, these systems are often misused and underutilized. The DriveID project aims to design and develop a passive, automated face identification system capable of robustly identifying the driver of the vehicle, retrieve a stored profile, and intelligently prescribing specific accident prevention systems and driving environment customizations.
\end{abstract}

Keywords: active safety, facial recognition, vehicle automation, teen fatalities, elderly drivers 


\section{Introduction}

The driving task is highly complex and places considerable perceptual, physical and cognitive demands on the driver. As driving is fundamentally an information processing activity, distracted or impaired drivers have diminished safety margins compared with non-distracted drivers $[7,25]$. This competition for sensory and decision making capacities can lead to failures that cost lives. Some groups, teens and elderly drivers for example, have patterns of systematically poor perceptual, physical and cognitive performance while driving. In 2009, about 3,000 teens in the United States aged 15-19 were killed and more than 350,000 were treated in emergency departments for injuries suffered in motor-vehicle crashes [3]. Teens are not only commonly novice drivers but they also have trouble maintaining attention and suffer from a greater propensity to engage in risky driving behaviors. Likewise, elderly drivers may also suffer from cognitive and sensory deficits which commonly lead to problems with visual acuity, change blindness, and reaction time deficits. Fatal crashes spike at 70 years of age [9].

At the macro level, it is clear that being in a vulnerable population leads to a greater risk of injury and death on the road. At the individual level, each crash can be attributed to poor judgment, slow responses, or failure to perceive cars or pedestrians. Each of these has its root in basic psychological and behavioral issues. Until recently, the complex nature of the relationship between psychology, behavior and driving was too poorly understood to effectively design systems that might intervene. The moment before a crash is difficult enough to understand physically, much less psychologically. As a result, safety systems have traditionally been somewhat fatalistic in their design; a bumper, airbag or child seat by its very nature assumes a collision. Recent advances in computational power, sensor technology, and the psychological and physiological understanding of workload within the driving environment are making possible a new, proactive generation of safety technology. Accident avoidance systems can now warn drivers of impending collisions, detect pedestrians, monitor drivers for microsleep events, and more. The driver's interaction with the environment can increasingly be augmented by the vehicle.

These advances are especially welcome in the case of vulnerable populations. The specific nature of the detriments to driving that these groups exhibit makes them, from an accident prevention system perspective, the low hanging fruit of safety. Accident avoidance systems targeted at teens include Tiwi, a tracking device capable of detecting speeding and cell phone use, and alerting parents by text message. Another contender is Ford's MyKey technology, in which specially made keys activate a speed governor system for novice drivers. Finally, a host of smartphone apps disable voice and texting when the GPS in the phone detects movement at vehicle speeds. The Elderly are served by MobilEye, a deep accident avoidance system including forward collision warnings, an advanced pedestrian detection system as well as lane departure and headway/tailway monitoring. These systems have the potential to save lives on a grand scale; NHTSA reported in 2005 that mandated traffic signal violation warnings alone had the potential to save over 17000 functional years of human life in the U.S. Stop sign violation warnings and in-vehicle signage systems together were projected to save an additional 3500 [16]. Despite these promising predictions, the penetration rates for accident avoidance systems are so low as to be unreported, even by manufacturers.

Why aren't accident avoidance systems that could save lives on such a scale in wider use? First, a number of pressures push designers toward designing for 'The General Public'. Even systems appropriate for the majority of drivers, such as airbags, may present dangers for specific subsections of the population [19]. The result is a reluctance to include population specific systems and devices, on the very real fear of the consequences involved. Additionally, even when such systems make it to market, disuse and abuse of systems [18] can hold them back from making an impact. While an accident avoidance system may assist one family member, a teen for example, it will increase workload for non-target family members using the vehicle. As a result, there must be a way to turn the system off. However, even the target group may not enjoy the system, and once turned off it often remains off. The system is therefore seen as inconsistent by the driver, the public, and by bodies attempting to evaluate the system's impact. Worst of all, the vulnerable party, and by extension the vulnerable population, is left unprotected. 


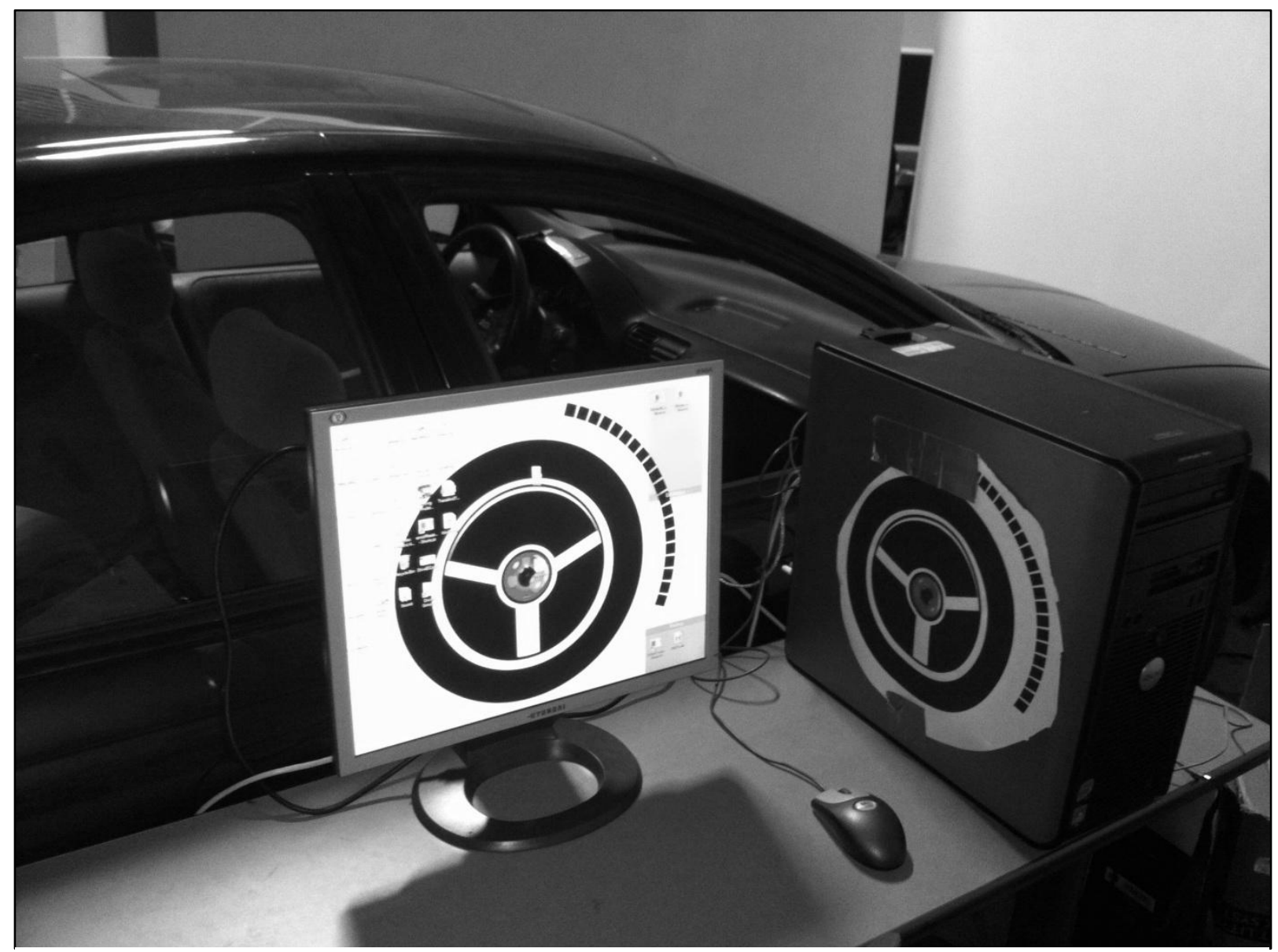

Figure 1: An experimental proving ground in the MIT2 Lab, where we are currently testing the pictured DriveID system under varying lighting and facial occlusion scenarios. Further information about the current generation of the system can be found at www.driveid.org.

\section{The Current Project: DriveID}

The DriveID project aims to design and develop a passive, automated face identification system capable of robustly identifying the driver of the vehicle, retrieve a stored profile, and intelligently prescribing specific accident prevention systems and driving environment customizations.

For example, upon entering her car, a teen named Mary would be identified despite her makeup and a new haircut. DriveID would retrieve her profile and engage a number of accident avoidance systems; a speed governor system capping Mary's roadway speed at $65 \mathrm{mph}$, a GPS enabled system designed to alert her mother should she use her phone while driving, and another designed to issue a text message alert when she arrived safely at school. As a finishing touch, mirror and seat systems would be in- structed to adjust to Mary, the climate control and radio would be set to her previous preferences, and the car would welcome her by name. From Mary's perspective the car would simply say "Welcome Mary.", and then drive as it should.

The system itself is compact, energy efficient, and inexpensive. It is based around a VGA webcam and compact CPU, and draws around 14 watts of power under peak usage. For reference, this is less than most in car GPS systems, but more than a dome light. The current system is freestanding and dashboard mountable, but could easily be integrated into a dashboard. DriveID runs custom software built using an SDK from Neurotechnology, a leading biometrics firm. We are proud and privileged to have Neurotechnology as DriveID's corporate sponsor.

\subsection{How We Addressed Safety}

The DriveID system ensures compliance as well as providing individuation and awareness benefits. 
Compliance is key to any successful safety system, asa reliable percentage of drivers will, quite literally, not flip a switch to save their lives. For instance, despite being mandatory and obviously beneficial, NHTSA reported seatbelt compliance nationwide to be about only $82 \%$ [5].The seatbelt, the only mandated safety system that requires a driver action to be effective, is through no coincidence the greatest battleground for safety in the modern vehicle. Bumpers, airbags and crumple zones are in part so successful because no driver action is required to ensure their safety contribution. By passively identifying drivers, DriveID allows accident avoidance systems to benefit from the same implicit compliance. DriveID automatizes compliance, allowing the driver to forget about the safety systems assisting them; the car simply greets them and protects them according to their needs.

Individuation is more than customization; it frees engineers and designers to help drivers in new and specific ways. DriveID allows for a targeted approach to safety, allowing designers to move beyond the 'General Public', evaluate vulnerable subgroups, and design accordingly. The result augmented driving environments that reduce or eliminate subgroups' detriments to driving, and resultant accidents. Drive ID also allows the flexibility to change the driving environment as easily as switching the driver. The ultimate vision is for individually tailored safety for all drivers of a vehicle.

Awareness refers to the advantages of having an intelligent automated system that knows the identities of each driver. Note that the majority of these advantages look beyond the core DriveID system, and instead relate to design opportunities the system creates within the vehicle. Take, for example, situations in which the driver is unknown by simply sending a picture and time stamp to the owner's phone whenever a new driver is detected, situations ranging from carjacking to friends driving your teen's car could be better managed. A number of in-vehicle systems, including OnStar, will call emergency services to the location of an accident. A DriveID enhanced system could also alert EMS personnel to the name and medical history of the driver. Longitudinal interventions are also possible: for example, by logging driving data for various users, the system could evaluate individuals' driving. It has been shown that in the absence of feedback, drivers tend to forget their roadway incidents very quickly, leaving their driving behavior unchanged. A study by Chapman and Underwood [4] found that an estimated $80 \%$ of nearaccidents are forgotten after 2 weeks. Accident avoidance systems focusing on education and awareness could be built around DriveID. The data could be given to insurance companies in return for lower rates. The system could suggest accident avoidance systems based on some driving patterns.

\section{Methodology}

\subsection{Participants}

Following the initial design phase of DriveID, a group of 9 participants were randomly selected and tested at the University of Central Florida. They were all treated in accordance with the American Psychological Association (APA) guidelines.

\subsection{Design and Procedures}

As the DriveID is essentially an identification system, a number of criteria were imposed to evaluate its efficacy. These criteria included:

- Accuracy (does it identify the correct person?)

- Reliability (does it do this consistently over time?)

- Robustness (does it do this consistently over various situations/lighting etc?)

- Speed of recognition

The dependent measures were (a) identification error rate and (b) ID recognition time, and (c) confidence level of the identification.

Nine individuals of different ages, gender and ethnicity participated in the evaluation. Each subject was first enrolled/registered into the DriveID. Subsequently, DriveID was tested to see if it recognized the individual successfully in a bright lighting condition (i.e. greater than $70 \mathrm{fcd}$ ), as well as low lighting condition (i.e. below $5 \mathrm{fcd}$ ). For each of these 2 lighting conditions, DriveID was tested with the following 5 facial orientations:

- Frontal view

- $\quad$ Slight tilt to the right (about 20 degrees)

- $\quad$ Slight tilt to the left (about 20 degrees)

- $\quad$ Slight tilt upward (about 20 degrees)

- $\quad$ Slight tilt downwards (about 20 degrees)

The design of the study assessing DriveID was a 2(level of lighting) X 5(angles of view) within subjects design. There were 3 trials for each of the 10 conditions. 


\subsection{Metrics used}

The DriveID was assessed on 3 criteria:

1. Accuracy: number of errors in identification

2. Identification time (in msecs)

3. Confidence level of identification

\section{Results}

The results of the evaluation of the DriveID in the 6 conditions are as follows.

\subsection{Dependent Measure: Percentage of Successful Identification}

\begin{tabular}{|l|l|l|l|l|l|}
\hline & $\begin{array}{l}\text { Full } \\
\text { Frontal }\end{array}$ & $\begin{array}{l}\text { Right } \\
20^{\circ}\end{array}$ & $\begin{array}{l}\text { Left } \\
20^{\circ}\end{array}$ & Up 20 & $\begin{array}{l}\text { Down } \\
20^{\circ}\end{array}$ \\
\hline $\begin{array}{l}\text { Bright } \\
\text { lighting } \\
\text { (Above } \\
70 \text { fc) }\end{array}$ & $100 \%$ & $66.67 \%$ & $88.89 \%$ & $66.67 \%$ & $66.67 \%$ \\
\hline $\begin{array}{l}\text { Low } \\
\text { lighting } \\
\text { (Below } \\
5 \text { fc) }\end{array}$ & $100 \%$ & $72.22 \%$ & $61.11 \%$ & $44.44 \%$ & $83.33 \%$ \\
\hline Overall & $100 \%$ & $69.45 \%$ & $75.00 \%$ & $55.56 \%$ & $75.00 \%$ \\
\hline
\end{tabular}

\subsection{Dependent Measure: Time to ID}

\begin{tabular}{|l|l|l|l|l|l|}
\hline & Front & Right & Left & Up & Down \\
\hline Bright & $\mathrm{M}=262.6$ & $\mathrm{M}=281.00$ & $\mathrm{M}=268.3$ & $\mathrm{M}=245.7$ & $\mathrm{M}=239.6$ \\
lighting & 7 & $\mathrm{SD}=0.47$ & 3 & 8 & 7 \\
(Above & $\mathrm{SD}=42.4$ & & $\mathrm{SD}=19.60$ & $\mathrm{SD}=97.8$ & $\mathrm{SD}=88.6$ \\
70 fc) & 4 & & & 0 & 2 \\
\hline Low & $\mathrm{M}=268.2$ & $\mathrm{M}=264.37$ & $\mathrm{M}=266.7$ & $\mathrm{M}=275.0$ & $\mathrm{M}=262.1$ \\
lighting & 8 & $\mathrm{SD}=26.57$ & 5 & 4 & 3 \\
(Below & $\mathrm{SD}=16.0$ & & $\mathrm{SD}=12.92$ & $\mathrm{SD}=6.86$ & $\mathrm{SD}=24.78$ \\
5 fc) & 8 & & & & \\
\hline Overall & $\mathrm{M}=265.4$ & $\mathrm{M}=272.69$ & $\mathrm{M}=267.5$ & $\mathrm{M}=260.4$ & $\mathrm{M}=250.9$ \\
& 8 & & 4 & 1 & 0 \\
\hline
\end{tabular}

\subsection{Dependent Measure: Confidence Level Ratings}

\begin{tabular}{|l|l|l|l|l|l|}
\hline & Front & Right & Left & Up & Down \\
\hline Bright & $\mathrm{M}=381.3$ & $\mathrm{M}=275.6$ & $\mathrm{M}=80.94$ & $\mathrm{M}=52.56$ & $\mathrm{M}=124.0$ \\
lighting & 3 & 7 & $\mathrm{SD}=30.50$ & $\mathrm{SD}=11.7$ & 0 \\
(Above & $\mathrm{SD}=234$. & $\mathrm{SD}=246$. & & 6 & $\mathrm{SD}=41.9$ \\
70 fc) & 46 & 54 & & 6 & 6 \\
\hline Low & $\mathrm{M}=697.3$ & $\mathrm{M}=103.5$ & $\mathrm{M}=101.79$ & $\mathrm{M}=60.96$ & $\mathrm{M}=113.4$ \\
lighting & 9 & 7 & $\mathrm{SD}=87.44$ & $\mathrm{SD}=19.9$ & 7 \\
(Below & $\mathrm{SD}=693$. & $\mathrm{SD}=92.0$ & & 1 & $\mathrm{SD}=42.0$ \\
5 fc) & 21 & 0 & & & 3 \\
\hline Overall & $\mathrm{M}=539.3$ & $\mathrm{M}=189.6$ & $\mathrm{M}=91.37$ & $\mathrm{M}=56.76$ & $\mathrm{M}=118.7$ \\
& 6 & 2 & & & 4 \\
\hline
\end{tabular}

\section{Discussion}

As long as a full frontal view of the face is available, DriveID can identify faces with near perfect accuracy. The software in inherently conservative, and software tests show a false acceptance rate (FAR) of less than $1 \%$. Accuracy, and correspondingly the confidence level, decreased at an angle. However, it is important to remember that, since time to identify faces appeared fairly consistent across all conditions, each attempt takes no more than 300ms. Assuming 2 seconds as a preferred window to make an identification, and 5 seconds as an outside window, DriveID has time for six to 15 attempts to establish a positive ID. During this time, it is unlikely that the driver will fail to look forward. Therefore, in most practical situations, DriveID will be working with a full frontal view.

Our team believes that how a product breaks can tell one a lot about how it works. In pursuit of that, we looked for the outer bounds of recognition. We can report that DriveID is robust to sunglasses, hair in the face, facial hair, objects in a driver mouth, behind their ears, makeup and more. It does have problems with occlusion of one eye, the entire mouth, and radical changes in a driver between drives, especially facial hair and glasses. However, once the system has recognized a driver in a new state, it will remain able to in the future. At around $30^{\circ}$ of rotation in any direction, DriveID can no longer recognize the driver's face. Around $.1 \mathrm{fcd}$ the low light cameras we use can no longer provide sufficient detail for recognition. Note that this is still sufficient for recognition on a lit street. Work is in progress on an infra-red version of DriveID. Preliminary results are promising, and a future system should be capable of identification in total darkness or by instrumentation lights.

\section{Conclusion}

We recently became national finalists and international semifinalist in NHTSA's 2011 Experimental Safety Vehicle Design Competition, coming out on top of over 200 other teams from the US. Our team then competed in Washington DC against eight other world-ranking teams representing some of the brightest student engineers of this generation. The company we kept was both humbling and vindication of the strength of the DriveID vision. We are now in the process of benchmarking, revising the code, reframing the technology based on what we learned, ans seeking new partners to join us in moving DriveID out of the lab and into the world. 


\section{References}

[1] Brovoid, S., Ward, N., Donath, M., \& Simon, S. (2007). Developing Driving Support Systems to Mitigate Behavioral Risk Patterns Among Teen Drivers. Report prepared for Intelligent Transportation Studies University of Minnesota, 511 Washington Avenue.

[2] Braitman, K.A., Kirley, B.B., McCartt, A.T., Chaushary, N.K. (2008). Crashes of novice teenage drivers: Characteristics and contributing factors. Journal of Safety Research, 39, 47-54.

[3] Centers for Disease Control and Prevention.Web-based Injury Statistics Query and Reporting System (WISQARS) [Online].(2010). National Center for Injury Prevention and Control, Centers for Disease Control and Prevention (producer).

[4] Chapman, P. \& Underwood, G. (2000). Forgetting nearaccidents: the role of severity, culpability and experience in the poor recall of dangerous driving situations. Applied Cognitive Psychology, 38, 215-227.

[5] Dempsey, J.S. \& Forst, L.S. (2007).An Introduction to Policing. Wadsworth Publishing

[6] Farmer, C.M. (2006). Effects of electronic stability control: an update. Traffic Injury Prevention, 7, 319-324.

[7] Hancock, P.A., \& Parasuraman, R. (1992).Human factors and safety in the design of intelligent vehicle-highway systems. Journal of Safety Research, 23, 181-198.

[8] Kahane, C. J. (1997). Relationships Between Vehicle Size and Fatality Risk in Model Year 1985-93 Passenger Cars and Light Trucks. NHTSA DOT HS 808570. http://www.nhtsa.dot.gov/cars/rules/regrev/evaluate/808570.ht $\mathrm{ml}$ (summary only).Washington, D.C.: U.S. Department of Transportation, National Highway Traffic Safety Administration.

[9] Levy, D.T., Vernick, J.S., \& Howard, K.A. (1995) Relationship Between Driver's License Renewal Policies and Fatal Crashes Involving Drivers 70 Years or Older. Journal of the American Medical Association, 274, 13. Retrieved Mar 26, 2011, from http://jama.ama-assn.org/content/274/13/1026.full.pdf

[10] McCartt, A.T., Hellinga, L.A., \&Haire, E.R. (2007). Age of licensure and monitoring teenagers' driving: Survey of parents of novice teenage drivers. Journal of Safety Research, 38, 697-706.

[11] NHTSA (2000).Traffic Safety Facts-Older Population. Report No. DOT-HS-809-328.US Department of Transportation, Washington DC.http://www-nrd.nhtsa.dot.gov/pdf/nrd30/ncsa/tsf2000/2000oldpop.pdf.

[12] NHTSA (2005a). Traffic Safety Facts: Speeding. 2004 Data. National Highway Traffic Safety Administration Report DOT-HS-809-915. US Department of Transportation, Washing DC.

[13] NHTSA (2006). Traffic Safety Facts Report No., DOT-HS809-919. US Department of Transportation, Washington DC.http://www.-nrd.nhtsa.dot.gov/pdf/nrd-

30/NCSA/TSFAnn/TSF2004.pdf
[14] NHTSA (2006). Traffic Safety Facts 2005 - Early Edition. National Highway Traffic Safety Administration Report Report No., DOT-HS-810-631. US Department of Transportation, Washington DC.

[15] NHTSA (2009).Fatality Analysis Reporting System (FARS), 2009. Washington, DC: U.S. Department of Transportation, National Highway Traffic Safety Administration, National Center for Statistics and Analysis.

[16] NHTSA (2005).Vehicle Safety Communications Project Task 3 Final Report.US Department of Transportation, National Highway Traffic Safety Administration.DOT-HS-809-859. March 2005.

[17] OECD (2006). Young Drivers: the road to safety. Organization of Economic Co-operation and Development and the European Conference of Ministries of Transport Report ITRD. OECD Publishing, Paris, France.

[18] Parasuraman, R., \& Riley, V. (1997). Humans and Automation: Use,Misuse, Disuse, and Abuse. Human Factors. 39 (2), pg.230-253.

[19] PhysOrg.com (2005).Airbags associated with increased probability of death in accidents, study finds. Retrieved Mar 28, 2011, from http://www.physorg.com/news4363.html

[20] Regan, M.A., Lee, J.D., \& Young, K.L. (2009). Driver Distraction: Theory, effects, and mitigation. CRC Press, Taylor and Francis, Boca Raton, FL, USA.

[21] Rinalducci, E.J., Smither, J.A., \& Bowers, C. (1993).The effects of age on vehicular control and other technological applications.In Wise, J.A., Hopkin, V.D., \&Strager, P. (Editors), Verification and Validation of Complex Systems: Additional Human Factors Issues. Daytona Beach, FL: Embry-Riddle University Press.

[22] Shinar, D. (2007).Traffic safety and human behavior. Amsterdam: Elsevier.

[23] Smith, E.E. (1968). Choice reaction time: An analysis of major theoretical positions. Psychological Bulletin, 69, 77100.

[24] Transportation Research Board, National Research Council. (1988a). Transportation in an aging society: Improving mobility and safety for older persons. Volume 1.Committee report and recommendations. Transportation Research Board Special Report 218. Washington, D. C.: National Academy of Sciences Press.

[25] Transportation Research Board, National Research Council. (1988b). Transportation in an aging society: Improving mobility and safety for older persons. Volume 2.Technical papers. Transportation Board Special Report 281. Washington, D.C.: National Academy of Sciences Press. 